ESJ Natural/Life/Medical Sciences

\title{
Facteurs Associés à L’altération de la Qualité de Vie Sexuelle Chez les Lombalgiques Chroniques à Cotonou
}

\author{
Zomalheto Z., \\ Possi S., \\ Dossou-Yovo H., \\ Hodjigue F.
}

Service de rhumatologie du Centre National Hospitalier Universitaire Hubert Koutoukou Maga de Cotonou

Doi:10.19044/esj.2021.v17n34p126

Submitted: 10 July 2021

Accepted: 10 August 2021

Published: 30 September 2021
Copyright 2021 Author(s)

Under Creative Commons BY-NC-ND 4.0 OPEN ACCESS

Cite As:

Zomalheto Z., Possi S., Dossou-Yovo H. \& Hodjigue F. (2021). Facteurs Associés à L'altération de la Qualité de Vie Sexuelle Chez les Lombalgiques Chroniques à Cotonou. European Scientific Journal, ESJ, 17(34), 126.

https://doi.org/10.19044/esj.2021.v17n34p126

\section{Résumé}

Objectif: Étudier les facteurs associés à l'altération de la qualité de vie sexuelle des patients souffrant de lombalgie chronique en milieu rhumatologique au Centre National Hospitalier et Universitaire Hubert Koutoukou Maga (CNHU-HKM) de Cotonou.

Patients et méthodes: Il s'est agi d'une étude transversale à visée descriptive et analytique qui s'est déroulée dans le service de rhumatologie du CNHU-HKM portant sur 127 patients lombalgiques chroniques chez qui la qualité de vie sexuelle a été évaluée grâce à une échelle de qualité de vie sexuelle validée (SQoL). Les données collectées ont été traitées et analysées à l'aide du logiciel Epi-info 7.2.1.0.

Résultats: La fréquence hospitalière des troubles de la sexualité était de $67,71 \%$. Les troubles sexuels retrouvés étaient majoritairement la baisse de la libido $(72,10 \%)$ et la diminution de la fréquence des rapports sexuels (59,30\%). La durée moyenne d'installation des troubles sexuels par rapport au début de la lombalgie était de 3 ans $\pm 2,70$. Soixante dix sept (77) patients (62\%) avaient une douleur lombaire per-coïtale. La majorité des patients (86\%) avait une mauvaise qualité de vie sexuelle dont 9 femmes sur 10 contre 3 hommes sur 4 . Le score SQoL moyen était de 49,13 $\pm 15,7$ [9-100]. Les 
facteurs associés à une altération de la qualité de vie sexuelle étaient le sexe féminin $(\mathrm{p}=0,022)$; la présence d'une irradiation $(\mathrm{p}=0,050)$; la baisse de la libido ( $\mathrm{p}=0,007)$ et l'absence de rééducation fonctionnelle dès le début de la lombalgie $(\mathrm{p}<0,001)$.

Conclusion: Au Benin, la qualité de vie sexuelle des patients lombalgiques est fortement altérée bien que le sujet soit tabou au sein de la population. Il importe donc au soignant d'inclure dans le but de la prise en charge des lombalgies chroniques l'amélioration de la qualité de vie pour une réponse plus efficace.

Mots clés : Qualité de Vie Sexuelle, Lombalgie chronique, Bénin

\section{Factors Associated with Impaired Quality of Sexual Life in Chronic Low Back Pain Patients in Cotonou}

\section{Dr. Karimou Laouali Idi}

Université Abdou Moumouni, Faculté des Sciences et Techniques, Département de géologie, Laboratoire de Géologie, Equipe Bassins et

Géoressources, Niamey, Niger

\section{Dr. Abdoulwahid Sani}

Université d'Agadez, Faculté des Sciences et Techniques, Département de

Géologie, Niamey, Niger

\section{Prof. Moussa Konaté}

Université Abdou Moumouni, Faculté des Sciences et Techniques, Département de géologie, Laboratoire de Géologie, Equipe Bassins et

Géoressources, Niamey, Niger

\section{Abstract}

Aim: To study the factors associated with the alteration of the sexual quality of life of patients suffering from chronic low back pain in a rheumatology setting at the Centre National Hospitalier et Universitaire Hubert Koutoukou Maga (CNHU HKM) in Cotonou.

Patients and methods: This was a descriptive and analytical crosssectional study conducted in the rheumatology department of the CNHUHKM on 127 patients with chronic low back pain whose sexual quality of life was assessed using a validated sexual quality of life scale (SQoL). The data collected were processed and analysed using Epi-info 7.2.1.0 software.

Result: The hospital frequency of sexual disorders was $67.71 \%$. The sexual disorders found were mainly a decrease in libido (72.10\%) and a decrease in the frequency of sexual intercourse (59.30\%). The average duration of the sexual problems in relation to the onset of the low back pain was 3 years \pm 2.70 . Seventy-seven (77) patients (62\%) had percoital low back 
pain. The majority of patients (86\%) had a poor quality of sexual life, including 9 out of 10 women and 3 out of 4 men. The mean SQoL score was $49.13 \pm 15.7$ [9-100]. Factors associated with an altered sexual quality of life were female gender $(\mathrm{p}=0.022)$; the presence of radiation $(\mathrm{p}=0.050)$; reduced libido ( $p=0.007$ ) and the absence of functional rehabilitation from the onset of low back pain $(\mathrm{p}<0.001)$.

Conclusion: In Benin, the sexual quality of life of patients with low back pain is strongly altered although the subject is taboo in the population. It is therefore important for the health care provider to include in the management of chronic low back pain the improvement of the quality of life for a more effective response.

Keywords: Quality of Sexual Life, Chronic Low Back Pain, Benin

\section{Introduction}

La lombalgie constitue un véritable problème de santé publique avec une incidence annuelle selon l'OMS de 60 à $90 \%$. De par ses multiples conséquences, elle a un impact sur la qualité de vie avec des répercussions psychosociales majeures qui peuvent affecter la sexualité (Bailly et al, 2015).

En Afrique, la sexualité reste un sujet tabou du fait de l'attachement des peuples aux valeurs morales et croyances religieuses (Pakpour et al, 2015) ce qui limite la prise en charge des patients souffrant des troubles sexuels et ne favorise point l'amélioration de leur qualité de vie. L'importance du bas du dos dans l'accomplissement de l'acte sexuel n'est plus à démontrer. L'atteinte d'une structure du rachis lombaire peut rendre douloureux l'acte sexuel favorisant une hypervigilance avec évitement de la douleur.

Cette hypervigilance entrainera l'apparition de troubles sexuels notamment une baisse de la libido (Bahouq et al, 2013). La détresse psychologique résultant de ces troubles constitue un facteur d'entretien de la lombalgie. Un cercle vicieux est donc constitué. L’objectif de ce travail a été d'étudier les facteurs associés à l'altération de la qualité de vie sexuelle des patients souffrant de lombalgie chronique en milieu rhumatologique au CNHU-HKM de Cotonou.

\section{Patients et méthodes}

\subsection{Type d'étude}

Nous avons conduit une enquête transversale de six (06) mois allant du 15 avril 2020 au 15 octobre 2020 dans le service de rhumatologie du CNHU-HKM pour mener une étude descriptive quantitative.

La population d'étude était constituée des patients lombalgiques qui ont consulté ou sont hospitalisés dans le service de rhumatologie. 
Sont inclus dans l'étude, les patients âgés d'au moins 18 ans, souffrant d'une lombalgie de plus de 3 mois et qui ont donné leur consentement par écrit ou verbalement.

Sont exclus de l'étude tout patient atteint de lombalgie inflammatoire ou infectieuse, tout patient ayant des troubles sexuels avant d'être lombalgique, tout patient sexuellement inactif pour des raisons autres que la lombalgie.

Pour collecter les données nous avions procédé à un entretien individuel structuré avec chaque patient au décours de leur consultation. Les informations étaient recueillies à l'aide d'une fiche d'enquête.

\subsection{Variables}

La variable d'intérêt était la présence ou non d'une bonne qualité de vie sexuelle. Elle a été évaluée à partir de la version francaise du Score de qualité de vie sexuelle Score Quality of Sex Life (SQOL-M) (Abraham et al, 2008) pour les hommes et SQOL-F pour les femmes (Symonds et al, 2005). Le score total de la qualité de vie se situe entre 0 et 100 (Abraham et al, 2008 ; Symonds et al, 2005). L'augmentation des scores se traduit par une meilleure qualité de vie sexuelle. Selon les données de l'étude de validation originale, les hommes et les femmes sans dysfonctionnement sexuel ont obtenu des scores moyens élevés pour la qualité de vie sexuelle $87,13 \%$ et $90,1 \%$ respectivement (Abraham et al, 2008 ; Symonds et al, 2005). Avaient donc une bonne qualité de vie sexuelle les sujets de sexe masculin et féminin ayant obtenu respectivement au moins $87 \%$ et $90 \%$ du score maximal avaient une bonne qualité de vie sexuelle.

Les variables indépendantes quant à elles étaient relatives aux caractéristiques sociodémographiques (age, sexe) ; au profil clinique (Indice de Masse Corporelle, intensité de la lombalgie, durée de la lombalgie, présence d'irradiation et atteintes de l'arc postérieure) et thérapeutique (traitement par antalgiques anti inflammatoires non stéroidiens, infiltrations lombaires aux corticoides, rééducation physique et enfin à l'activicté sexuelle (présence ou non de troubles sexuels).

\subsection{Analyse des données}

Les données ont été traitées et analysées à l'aide du logiciel Epi-info 7.2.1.0. Les mesures descriptives ont été utilisées pour synthétiser les données. Les associations entre la variable dépendante et les différentes variables indépendantes ont été étudiées avec les tests de Chi2 ou test exact de FISHER. Le seuil de significativité est de 5\%, p-valeur $<0,05$. 


\subsection{Difficultés et limites de l'étude}

La difficulté rencontrée au cours de ce travail était la réticence de certains patients à décrire leur habitude sexuelle. Une mise en confiance des enquétés a permis de surmonter cette dernière.

\section{Résultats}

\subsection{Caractéristiques générales des patients}

L’âge moyen des 127 (87 hommes, 40 femmes) patients était de 49,83 ans $\pm 11,77$ [extrêmes: $19-80$ ans ] avec un sex-ratio $(\mathrm{H} / \mathrm{F})$ de 0,46.

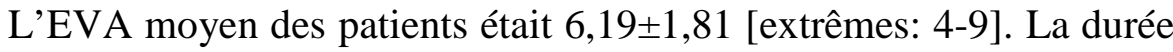

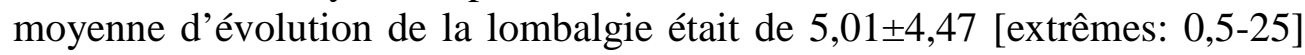
ans. L'irradiation dans un membre pelvien était observée chez 78 patients (61,40\%). L'atteinte de l'arc antérieur était présente chez 99 patients (77,95\%).

Le tableau I résume les caratéristiques générales des patients.

Tableau I. Caractéristiques générales des patients

\begin{tabular}{|c|c|}
\hline & Effectif (n=127) \\
\hline & Moyenne \pm SD ou n (\%) \\
\hline Caractéristiques socio démographique & 0,46 \\
\hline Sex ratio (H/F) & $49,83 \pm 11,77[19-80]$ \\
\hline Age moyen (ans) & \\
\hline Caractéristiques cliniques & $28,96 \pm 5,54[18-46,38]$ \\
\hline IMC (kg/m²) & $6,19 \pm 1,81[4-9]$ \\
\hline Intensité de la lombalgie & $5,01 \pm 4,47[0,5-25]$ \\
\hline Durée de la lombalgie (en années) & $78 / 127(61,40 \%)$ \\
\hline Présence d'irradiation & $99 / 127(77,95 \%)$ \\
\hline Atteinte de l'arc antérieur & $127 / 127(100 \%)$ \\
\hline Caractéristiques thérapeutiques & $113 / 127(89 \%)$ \\
\hline Antalgiques & $79 / 127(62 \%)$ \\
\hline AINS* & $49 / 127(39 \%)$ \\
\hline Myorelaxants & $44 / 127(35 \%)$ \\
\hline Infiltration rachidienne corticoïdes & \\
\hline Rééducation fonctionnelle & \\
\hline Anti Inflammatoire Non Stéroïdien & \\
\hline & \\
\hline
\end{tabular}

\subsection{Activité sexuelle des patients}

Quatre vingt-six patients $(67,71 \%)$ avaient des troubles de la sexualité. La durée moyenne d'installation des troubles sexuels après le début de la lombalgie était de $3 \pm 2,77$ [1-20] ans. Ces troubles étaient plus fréquents chez les sujets de sexe féminin ( $\mathrm{n}=87 ; 68,5 \%)$. La baisse de la libido était le trouble sexuel le plus rencontré $(72,10 \%)$ suivi de la diminution de la fréquence des rapports sexuels $(59,30 \%)$. 
Les douleurs lombaires au cours des rapports sexuels (douleur lombaire percoitale) étaient présentes dans $62 \%$ des cas. enquêtés.

La figure 1 retrace les différents troubles sexuels des patients

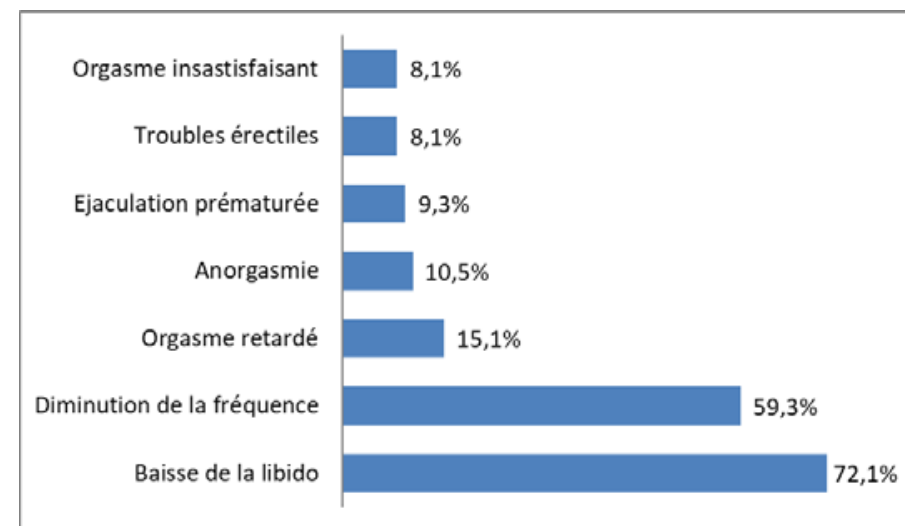

Figure 1. Différents troubles sexuels des patients

\subsection{Qualité de vie sexuelle des patients}

Selon les données de l'étude de validation du score SQoL avaient une bonne qualité de vie sexuelle les sujets de sexe masculin et féminin ayant obtenu respectivement au moins $87 \%$ et $90 \%$ du score maximal avaient une bonne qualité de vie sexuelle.

Tableau II. Qualité de vie sexuelle des patients

\begin{tabular}{|c|c|}
\hline & Effectif (n=127) \\
\hline & Moyenne \pm SD ou n (\%) \\
\hline Prévalence de la mauvaise qualité de vie & $109 / 127(85,80 \%)$ \\
\hline Score SQoL moyen & $49,13 \pm 15,70[9,09-100]$ \\
\hline Homme & $42 \pm 14,59[11-66]$ \\
\hline Femme & $50,14 \pm 15,9[18-100]$ \\
\hline
\end{tabular}

\subsection{Facteurs associés à l'altération de la qualité de vie sexuelle}

Le sexe féminin, la présence d'une irradiation au membre pelvien, la présence des troubles sexuels et l' absence de la rééducation fonctionnelle étaient statistiquement associés à une altération de la qualité de vie sexuelle $(\mathrm{p}=0,022, \mathrm{p}=0,050, \mathrm{p}=0,007$ et $\mathrm{p}=0,001$ respectivement). Les femmes avaient un risque élevé de $70 \%$ d'avoir une mauvaise qualité de vie sexuelle comparé aux hommes et les sujets ayant des troubles avaient un risque multiplié par quatre (4) d'avoir une mauvaise qualité de vie sexuelle.

Parmi les troubles sexuels, seule la baisse de la libido était statistiquement associée à une altération qualité de vie sexuelle $(\mathrm{p}=0,021$; $\mathrm{OR}=3,98$; IC : 1,33-14,74). 
Par contre le massage du dos avant les rapports sexuels apparait comme un facteur d'une bonne qualité de vie sexuelle $(\mathrm{p}=0,047$; OR=0,19 ; IC : 0,03-0,86) et réduisait de $81 \%$ le risque d'avoir des crises douloureuses lombaires pendant les rapports sexuels.

Le tableau III met en évidence les différents facteurs associés à l'altération de la qualité de vie sexuelle.

Tableau III. Facteurs associés à l'altération de la qualité de vie sexuelle

\begin{tabular}{|c|c|c|}
\hline Facteurs associés & P-value & OR (IC) \\
\hline Sexe féminin & 0,022 & $0,30(0,11-0,84)$ \\
\hline Présence d'irradaition & 0,050 & $0,27(0,06-0,89)$ \\
\hline Baisse de la libido & 0,021 & $3,98(1,33-14,74)$ \\
\hline Absence de réeducation fonctionnelle & 0,001 & $0,10(0,03-0,30)$ \\
\hline Massage du dos avant les rapports sexuels & 0,047 & $0,19(0,03-0,86)$ \\
\hline
\end{tabular}

\section{Discussion}

La prédominance féminine des patients lombalgiques notée dans notre étude pourrait être liée à certains facteurs prédisposant dans la gent féminine telles que les grossesses, les activités ménagères. Mais, dans la littérature il n’y a pas une unanimité par rapport à ce résultat (Ambler et al, 2001).

La prévalence des troubles de la sexualité était faible par rapport aux données de Bahouq et al (2013) qui étaient de 81\% (Bahouq et al, 2013). Cette prévalence élevée témoigne de l'impact négatif de la lombalgie sur la sexualité.

La prédominance masculine des troubles de la sexualité s'expliquerait par la manifestation plus expressive sur le plan physique des troubles de la sexualité chez l'homme en comparaison avec celle de la femme ; l'acte sexuel chez l'homme étant avant tout conditionné par la qualité de l'érection (Sjogren et al, 1981).

La baisse de la libido et la diminution de la fréquence des rapports sexuels étaient également retrouvées par Maigne (2001) en France.

Par ailleurs, la forte prévalence de la douleur lombaire per coïtale constatée également chez Bahouq et al en 2013 témoigne également de l'handicap que représente la lombalgie pour la réalisation de l'acte sexuel.

La qualité de vie sexuelle était fortement altérée chez les patients avec un score SQoL moyen qui avoisine celui de Bahouq et al (2013) au Maroc $(44,65 \pm 17,4)$. Cette altération s'expliquerait par la prévalence élevée des troubles sexuels et de la douleur lombaire per coïtale dans notre population.

Le sexe féminin, la présence d'une irradiation au membre pelvien, la présence des troubles sexuels et l'absence de la rééducation fonctionnelle étaient statistiquement associés à une altération de la qualité de vie sexuelle (respectivement $\mathrm{p}=0,022, \mathrm{p}=0,050, \mathrm{p}=0,007$ et $\mathrm{p}=0,001$ ). 
Le handicap lié à la douleur lombaire pourrait expliquer que les femmes avaient plus de risque d'avoir une mauvaise qualité de vie sexuelle car elles souffriraient de douleur plus souvent et plus longtemps que les hommes (Maigne et al, 2001).

La mauvaise qualité de vie sexuelle n'était pas asssociée à l'âge dans notre serie. Bahouq et al (2013) par contre, notait que la qualité de vie sexuelle était plus pertubée chez les patients agés $(\mathrm{p}=0,003)$ du fait qu'au fil des années, la tonicité physiologique diminue de même que l'intensité et la qualité de la relation sexuelle (Bahouq et al, 2013). Par ailleurs, Il est généralement admis que les patients âgés ont moins d'activité sexuelle que les jeunes (Sjogren et al, 1981).

Au plan médical, la rééducation du rachis lombaire axée sur la physiothérapie sédative, les massages doux pourraient permettre d'améliorer la qualité de vie sexuelle des patients $(\mathrm{p}=0,047$; OR=0,19; IC : 0,03 0,86 ) comme l'ont démontré d'autres auteurs (Alagnide et al, 2017; Pynor et al, 2005) .

\section{Conclusion}

Les troubles sexuels restent fréquents au cours des lombalgies communes. L’impact de la lombalgie sur la qualité de vie sexuelle et l'altération significative des différentes étapes de la réponse sexuée restent certaine. La prise en charge de toute lombalgie doit prendre en compte cette qualité de vie pour une action optimale sur l'allègement de la souffrance du patient. La rééducation semble avoir un effet positif qu'il faudra confimer par des études cas témoins. Par ailleurs, une prise en charge psychologique doit nécessairement faire partie de l'arsenal thérapeutique de toute lombalgie chronique pour une amélioration de la qualité de vie sexuelle des patients.

\section{References:}

1. Frymoyer JW, Cats-Baril WL. An overview of the incidences and costs of low back pain. Orthop Clin North Am. 1991;22(2):263-71.

2. Bailly F, Foltz V, Rozenberg S, Fautrel B, Gossec L.Les répercussions de la lombalgie chronique sont en partie liées à la perte du rôle social: une étude qualitative.Rev Rhum.2015; 82(6):396-401.

DOI:10.1016/j.rhum.2015.08.005.

3. Pakpour AH, Nikoobakht M, Campbell P. Association of pain and depression in those with chronic low back pain: the mediation effect of patient sexual functioning. The Clinical journal of pain. 2015;31(1):44-51.

DOI:10.1097/AJP.0000000000000076. 
4. Bahouq H, Fadoua A, Hanan R, Ihsane H, Najia H-H. Profile of sexuality in Moroccan chronic low back pain patients. BMC Musculoskeletal Disorders.2013;14(1):63.

DOI: 10.1186/1471-2474-14-63.

5. Abraham L, Symonds T, Morris MF: Psychometric validation of a sexual quality of life questionnaire for use in men with premature ejaculation or erectile dysfunction. The SQOL-M J Sex Med 2008, 5:595-601.

6. Symonds T, Boolell M, Quirk F: Development of a questionnaire on sexual quality of life in women. J Sex Marital Ther 2005, 31:385-397.

7. Ambler N, de C, Williams AC, Hill P, Gunary R, Cratchley G. Sexual difficulties of chronic pain patients. Clin J Pain.2001;17(2):138-45. DOI:10.1097/00002508-200106000-00006.

8. Sjogren K, Fugl-Meyer AR. Chronic back pain and sexuality. International rehabilitation medicine. 1981;3(1):19-25. DOI:10.3109/03790798109167110.

9. Maigne J-Y, Chatellier G. Assessment of sexual activity in patients with back pain compared with patients with neck pain. Clinical Orthopaedics and Related Research.2001;(385):82-7.

DOI: 10.1097/00003086-200104000-00014.

10. Lopes P, Poudat F-X. Manuel de sexologie. 2ème édition. Paris: Elsevier Masson;2010.

11. Tousignant-Laflamme Y, Marchand S. Sex differences in cardiac and autonomic response to clinical and experimental pain in LBP patients. Eur J Pain 2006, 10603-614. DOI: 10.1016/j.ejpain.2005.09.003.

12. Alagnide HE, Houngbedji GM, Azanmasso $\mathrm{H}$ et al. Résultats du traitement rééducatif des lombalgies suivies au Centre National Hospitalier et Universitaire Hubert Koutoukou Maga de cotonou. J. Rech. Sci. 2017; 19(3):413-22.

13. Pynor R, Weerakoon P, Jones MK et al. A preliminary investigation of physiotherapy students attitudes towards issues of sexuality in clinical practice.Physiotherapy.2005;91(1):42-8.

DOI:10.1016/j.physio.2004.06.012. 